\title{
A STATISTICAL INVESTIGATION OF MICROWAVE BURST SPECTRA FOR THE DETERMINATION OF SOURCE INHOMOGENEITIES
}

\author{
W. SCHOECHLIN AND A.MAGUN \\ Institute of Appliea Physics \\ University of Berne \\ Sidlerstrasse 5/3012 Berne \\ Switzerland
}

Summary. The spectra of a set of 77 microwave bursts of July 1971 to September 1974 as published in Solar Geophysical Data have been fitted with an idealized spectrum of triangular shape. The spectral index of the low frequency part $\alpha$, the maximum flux density and the associated frequency have been determined. A cluster-analysis carried out on these parameters showed that the sample consists of only one class of events. In the histogram of the spectral index we found a most frequent value of 1.4. Such a low value cannot be explained by the mechanisms normally assumed to account for the low frequency attenuation, as gyroresonance absorption and the Razin effect.

In order to explain this discrepany we introduced a model with an idealized thermal source of cylindrical shape. The magnetic field and the source temperature decay radially $(R)$ and slowly, following a powerlaw with exponents $\boldsymbol{\gamma}_{\boldsymbol{B}}$ and $\boldsymbol{\gamma}_{\boldsymbol{T}}$ outside a homogeneous center with radius $R_{0}$ and temperature $T_{0}$. Taking into account only the radiation of the optically thick part, which extends from the source center to radius $R_{T}$, the observed flux can be written in the Rayleigh-Jeans approximation as:

$$
S(f)=.16 f^{2} \int_{0}^{R_{T}(f)} T(R) 2 \pi R d R
$$

The absorption coefficient of gyrosynchrotron radiation is increasing with decreasing frequency. Therefore outer parts of the source become optically thick and the source radius increases. With the frequency below which thermal gyrcsynchrotron radiation becomes self absorbed (Dulk et al., 1978) and our source model the relationship between $R_{T}$ and $f$ was obtained and the integration carried out:

$$
\begin{aligned}
& S(f)=.16 \pi R_{0}^{2} T_{0} f^{2-\delta} \text {, where } \\
& \delta=\frac{2\left(f / f_{0}\right)^{-E}}{2\left(f / f_{0}\right)^{-E}-\gamma_{T}^{g}} \cdot E \text {, and } E=\frac{2-\gamma_{T}^{2}}{\partial_{B}^{2}+.5 \gamma_{T}^{2}}
\end{aligned}
$$

The calculated dependence of the spectral index $\alpha=2-\delta$ on the exponents $\partial_{T}$ and $\partial_{B}^{e}$ is shown in Figure 1 . From this diagram follows, that a 183

M.R. Kundu and T.E. Gergely (eds.). Radio Physics of the Sun, 183-18.5.

Copyright $\odot 1980$ by the IAU. 
spectral index of 1.4 (the most frequent value observed) is due to a source, where the parameters reach half their central values at about twice the radius of the central region.

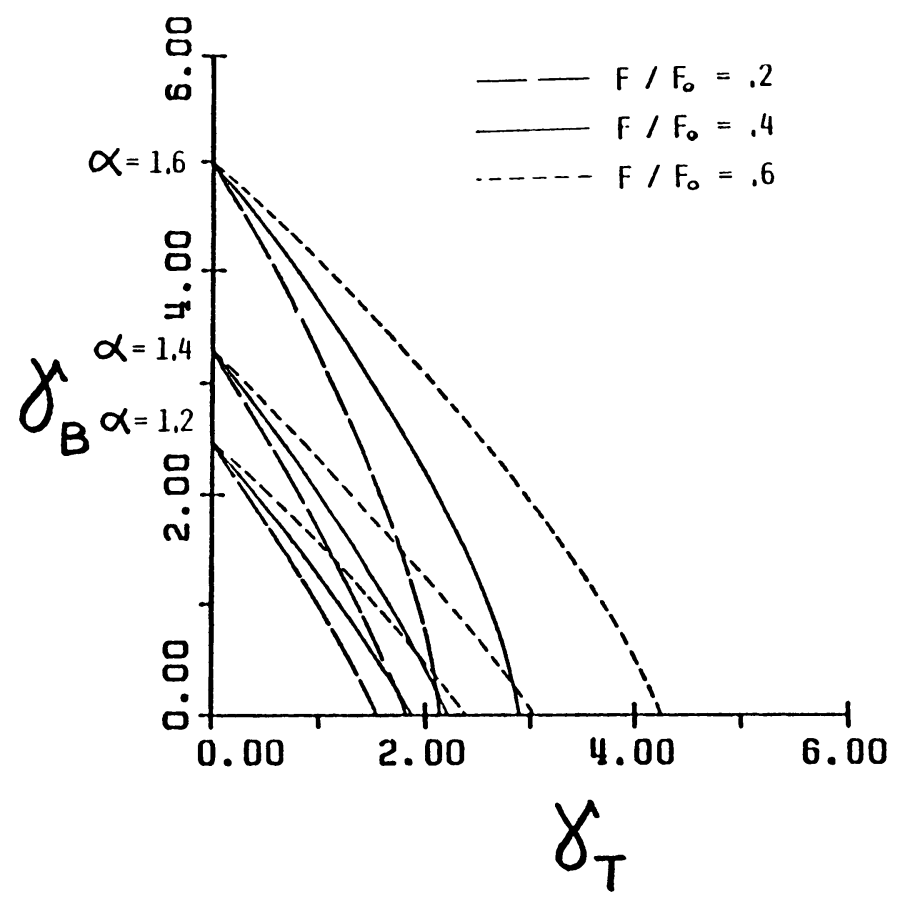

FIGURE 1. Graph, showing possible values of $\boldsymbol{\gamma}_{\mathcal{T}}$ and $\mathcal{\gamma}_{\boldsymbol{B}}$ for spectral in-

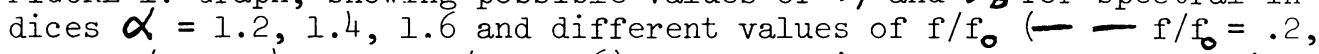
$\left.\mathrm{f} / \mathrm{f}_{0}=.4,---\mathrm{f}_{\mathrm{f}} \mathrm{f}_{0}=.6\right)$, where $\mathrm{f}_{0}$ is the frequency at which the center of the source becomes optically thick.

Also a correlation analysis has been carried out on the parameters defined at the beginning, supporting our hypothesis that inhomogeneities in temperature and magnetic field determine the different spectral indices in different events and showing that the large differences in the maximum flux in different events are mainly produced by different va.lues of temperature and magnetic field.

\section{REFERENCE}

Dulk G.A., Melrose D.B., and White S.M., 1978, to be published in Ap.J. ACKNOWLEDEGEMENT

This work was supported by the Swiss National Science Foundation (NF) Under grant No. 2.628-0.76. 


\section{DISCUSSION}

Trehan: Is it physically realizable that you have a constant radial magnetic field inside a cylindrical geometry? A potential field outside seems all right. In any case, I suspect that the magnetic field configuration you suggest will have a surface current which may lead to instabilities. If you are talking of the magnetic field along the Z-axis, then a $1 / \mathrm{r}$ field outside the cylinder gives a $j \alpha \frac{l}{r}$ and this is again unrealistic. In the first case the divergence equation is not satisfied in the inside region.

Schoechlin: Our picture is an idealization of the physical situation. Inhomogeneiteies in the central part are not considered, as they are not relevant to the spectral index. Any decrease of the parameters outside the source will cause the spectral index to decrease below two.

Vlahos: Is your configuration stable to MHD instabilities or currents which can develop due to the steep magnetic field grandients?

Schoechlin: Examples for a magnetic field structure with "steep" field gradients at the boundary are loops. They are stable over much longer times than the duration of impulsive microwave bursts. 